\title{
Influence of Light Polarization on Reorientation Nonlinearity in Nematic Liquid Crystals
}

\author{
K.A. BRZDAZKIEWICZ* AND M.A. KARPIERZ \\ Faculty of Physics, Warsaw University of Technology \\ Koszykowa 75, 00-662 Warsaw, Poland
}

\begin{abstract}
Nematic liquid crystals are characterized by a giant optical nonlinearity which has its origin in the molecular reorientational phenomenon. Uniqueness of this nonlinear mechanism causes that response of the medium is dependent on many conditions. Among various circumstances, both an initial alignment of liquid crystal cell and geometry of the system are very important, but in this paper a significant role of light polarization in nonlinear effects observed in nematics is emphasized. Additionally, it is underlined that dependence on light polarization does not have only trivial aspect of initial conditions. More complex seems to be the fact that due to the high optical anisotropy of the medium the state of light polarization is changing on the way of beam propagation. As a consequence, the modifications of molecular orientation in the liquid crystal cell are strongly dependent upon light polarization.
\end{abstract}

PACS numbers: 42.65.-k, 42.70.Df

\section{Introduction}

The nonlinear optics of liquid crystals (LCs) is an area of intensive studies for more than two last decades. Physical basis describing properties of LCs are well known, shown and widely discussed in numerous publications, papers and monographs [1-5]. Many groups of researchers and theoreticians concentrate their work on nonlinear effects in nematic liquid crystals (NLCs) caused by molecular reorientation phenomenon. The latter has been investigated theoretically and demonstrated experimentally in a great number of system's geometries, i.e. for different LC mesophases and textures, different light polarizations, different angles of beam's incidence, and finally with and without additional assistance of exter-

*corresponding author; e-mail: kasia@if.pw.edu.pl 
nal field(s). In this paper one of the important aspects of the problem, namely, dependence of nonlinear response on light polarization is shown and discussed. This subject is not innovative or novel one and has been already studied in the cases of linear [6-18], circular [19-23] and elliptical [24-26] polarization as well as in the case of completely non-polarized light [26, 27]. All performed experiments demonstrate that the beam polarization can be used as an effective parameter to control the LC reorientation which gives rise to some very unique nonlinear effects that can appear for one state of polarization and disappear for the other ones. Well known optical Freedericks transition (OFT) was the first example reported in the literature [1-12,28], but many other unusual optical effects were discovered in the last fifteen years, such as self-induced stimulated light scattering [19, 20, 25], optical bistability, discontinuities and hysteresis $[19,14]$, nonlinear optical oscillations [13, 21], spontaneous and unstable spatial pattern formation [15, 16], bifurcations with further transitions toward an irregular regime $[17,18,22,23]$ and deterministic chaos [14], etc.

\section{Theoretical analyses}

Let us introduce a unit vector $n$ called as director. It defines average molecular alignment in the $\mathrm{LC}$ cell and its direction corresponds to the mean position of the long axes of molecules. Without influence of any of external field, molecules fluctuate around the average arrangement given by director. If external field(s) is/are applied, the orientation inside the cell is changing and the new ordering is obtained as a result of system's free energy minimization [1-5]. From macroscopic point of view most of LCs are positive uniaxal materials with optical axis corresponding to the ordering direction described by director $\boldsymbol{n}$. It means that, when electric field of the propagating light wave tends to align the LC molecules along $\boldsymbol{E}$ vector, the birefringence axis is rotated. This is a mechanism of reorientational nonlinearity which is typical of LCs and has a lot of properties that are unattainable in cases of other types of optical nonlinearities.

Nonlinear properties connected with reorientation are strongly dependent on molecular arrangement and therefore they depend on LC cell configuration. In particular, nonlinear characteristics can be fitted to required form by special preparing of the initial orientation in the sample. Commonly known fact is that when the light polarization is linear and direction of electric field is parallel to director $n$, non-reorientation effect appears but in other cases it occurs generally and additionally process of molecular reorientation could have a threshold character (when electric field is orthogonal to director).

Let us analyze an influence of the initial cell orientation and light polarization on nonlinearity, basing on an example of light beam which is linearly polarized in $x$ direction and it illuminates LC cells shown in Fig. 1. For twisted alignment (c) reorientation takes place for any value of power while for homeotropic texture 


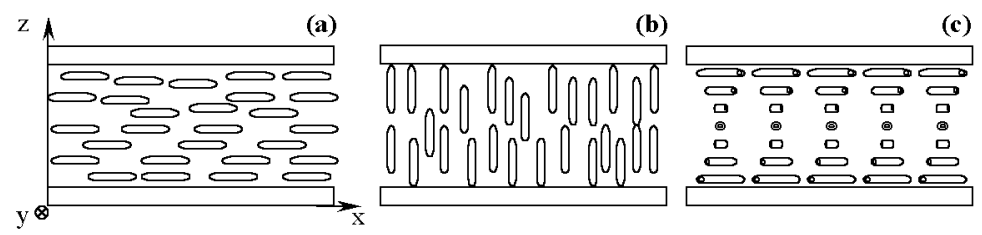

Fig. 1. Three various types of basic nematic liquid crystal cells configuration: (a) planar, (b) homeotropic, (c) twist.

(b) this process has threshold character. In case of planar configuration (a) LC layer appears as a linear medium for the light beam illuminating a cell because $E_{x}$ component of electric field is not able to reorient molecules.

But the dependence on light polarization does not have only this trivial aspect of initial conditions. Much more complex is the fact that due to the high optical anisotropy of LCs (with a typical value of 0.2 for nematics in the visible region), the state of light polarization is changing inside the sample. Self-induced polarization rotation in liquid crystal medium is not a new optical phenomenon and it has been reported in many papers where it is shown as a result of angular momentum transfer from the optical field to the medium, causing molecules to rotate around the beam propagation direction $[19,20,25]$. The first experimental observation of such effect in nematic layer is presented in Ref. [19] where the rotation of molecules is manifested by rotation of the polarization ellipse of the output beam. Also in other publications one can find next evidences that there exists interplay between optically induced molecular reorientation and electric field of the light wave. It leads to generation of different polarization states and thus to various optical effects and nonlinear dynamical behaviors [25, 26, 13-18].

To make mathematical description easier, it is useful to introduce another variable, i.e. reorientation angle $\theta$. Let us define it as an angle between director $n$ and $x$-coordinate. Angle $\theta$ is fully sufficient to describe reorientation process in 2D problems, i.e. when molecules and vector of influent electric field are in the same plane.

In all theoretical investigations and also in experimental part of this work planar nematic liquid crystal cell (shown in Fig. 1a) was used. It is characterized by identical conditions on both boundaries and moreover it can be assumed that initially reorientation angle $\theta$ is the same in the whole volume of the sample. In analyzed cases $\theta$ is equal to zero when non-external field is applied.

Let us consider physical setup from Fig. 2a. Linearly polarized electromagnetic wave with vector $\boldsymbol{E}$ is incident orthogonally on the planar cell. In nonlinear regime, LC molecules are forced to be reoriented in $x-y$ plane. Angle $\beta$ (introduced as the angle between electric field direction and $x$-coordinate) defines the ratio between $E_{x}$ and $E_{y}$ components of electric field intensity vector. Figure $2 \mathrm{~b}$ shows the schematic plot of director reorientation process as a result of electric field interaction. 


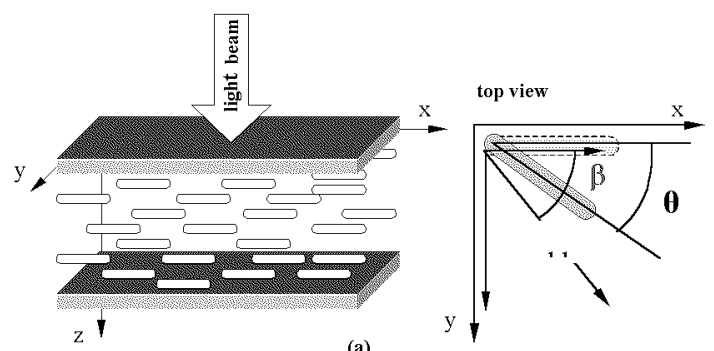

(b)

Fig. 2. Configuration of analyzed system: (a) planar cell illuminated by orthogonally incident light beam; (b) schematic drawing of reorientation process taking place in $x-y$ plane as an effect of electric field influence.

In the input plane (i.e. $z=0$ ) reorientation angle $\theta=0^{\circ}$ and the angle $\beta$ can be taken as a variable value describing direction of influent field. Of course, for different $\beta$ there are individual initial conditions and it causes that different distribution of reorientation angle inside the cell is obtained.

In a case of an infinite plane wave with $E_{y}$ and $E_{x}$ components, reorientation of the liquid crystal molecules (i.e. distribution of reorientation angle $\theta$ ) can be calculated from differential Euler-Lagrange equation, which specifies minimization of the total free energy of the system and could be written in the following form [1]:

$$
K_{22} \frac{\partial^{2} \theta}{\partial z^{2}}+\frac{\varepsilon_{0} \Delta \varepsilon}{4}\left[\left(\left|E_{y}\right|^{2}-\left|E_{x}\right|^{2}\right) \sin 2 \theta+\left(E_{x}^{*} E_{y}+E_{x} E_{y}^{*}\right) \cos 2 \theta\right]=0,
$$

where $\varepsilon_{0}$ is the permittivity of free space, $\Delta \varepsilon=n_{\mathrm{e}}^{2}-n_{\circ}^{2}$ is the electrical anisotropy, and $K_{22}$ is the Frank (elastic) constant corresponding to the twist deformation in NLCs.

It is worth noting that results of all numerical simulations presented in this paper were obtained for parameters characteristic of 6CHBT nematic liquid crystal, i.e. $n_{\mathrm{o}}=1.52, n_{\mathrm{e}}=1.69, K_{22}=3.5 e-12 \mathrm{~N}$ and for wavelength of $514 \mathrm{~nm}$. Moreover, in all theoretical calculations strong anchoring boundaries conditions were assumed. It means that molecules are rightly located at boundaries surfaces and never change their orientation even in case of very high power of influent external field.

Analyses of reorientation process inside liquid crystal cell are often simplified by introducing various approximations. Figure $3 \mathrm{a}$ shows typical reorientation distribution obtained by numerical solution of differential Eq. (1) for $\beta=45^{\circ}$ with an assumption that the state of light polarization is the same along thickness of the cell. As one can see, curves have a form from nearly sinusoidal for low powers, up to square-like distribution in case of the high power which causes that molecules line up in the direction of electric field in nearly whole sample. This kind of analyses is still employed [6-9] and even if they lead to correct results and conclusions, they should be taken somehow as theoretical oversimplification because in real sit- 


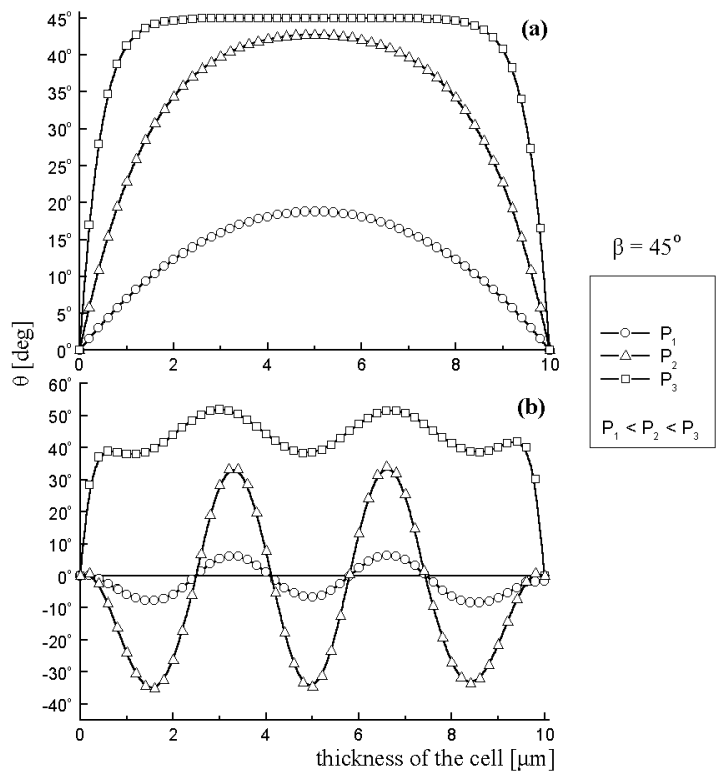

Fig. 3. Longitudinal reorientation distribution in planar nematic liquid crystal cell obtained by numerical solutions of Eq. (1) for $\beta=45^{\circ}$ and cell thickness of $10 \mu \mathrm{m}$ : (a) with assumption that state of polarization does not change while light is passing through the cell, (b) after full analyses of light polarization state inside the medium.

uations the state of polarization is changing when the light is passing through LC sample and initially vector $\boldsymbol{E}$ is not parallel to optical axis. This fact can be easily demonstrated by analyzing polarization ellipticity and azimuth inside the traditional uniaxal, birefringent crystal. The spatial period of obtained characteristics (calculated for $\theta=0^{\circ}, \lambda=514 \mathrm{~nm}$ and $\Delta n=0.17$ ), shown in Fig. $4 \mathrm{a}$,b, can be described by a birefringence length $L_{\mathrm{B}}=\lambda / \Delta n$ and it reflects exactly the changes in polarization state along the medium. As it is expected, the direction of long axis of polarization ellipsoid described by azimuth is rotating in the sample. Moreover, the state of light polarization is changing continuously from linear (ellipticity =1) through elliptical to circular (ellipticity $=0$ ). Range of these changes depends on the value of angle $\beta$ which defines here the angle between initial direction of electric field and birefringence axis.

In contrast to the previous, simplified case shown in Fig. 3a, reorientation profiles obtained with theoretical assumptions including light polarization aspects are totally different. Namely, the change of polarization inside LC cell induces periodical spatial modulation of reorientation angle $\theta$ which is shown in Fig. 3b. These results are in agreement with the intuitive predictions, basing on results illustrated by Fig. 4 and they demonstrate that LC molecules are rotating with spatial period corresponding to the birefringence length. 


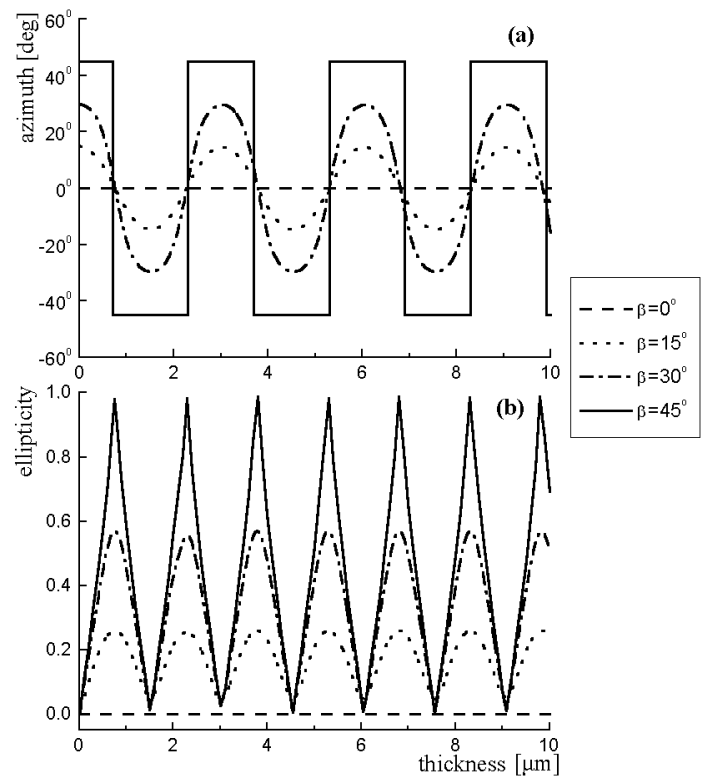

Fig. 4. Theoretical analyses of polarization state changes inside the birefringent medium characterized by $n_{\mathrm{e}}-n_{\mathrm{o}}=0.17$. Azimuth (a) and ellipticity (b) of the optical beam with a wavelength of $514 \mathrm{~nm}$ was calculated for different values of angle $\beta$ which is an angle between birefringence axis and initial linear polarization of the beam.

Statement that molecular reorientation in LC cell has to be evaluated at once with Maxwell's equations has appeared in literature in early 80's. First theoretical models proposed by Zel'dovich [10,11] and Ong [12] describe only the simple cases of system's geometries in which light polarization remains linear inside reoriented sample. In Ref. [28] it is underlined that for linearly polarized input beam it is necessary to consider the change of the beam polarization state inside LC cell due to birefringence taking its origin from molecular reorientation. In further works of Santamato et al., Hamiltonian formulation of the light propagation for the case of normal incidence and simplified expression for reorientation is presented [24] and it is expanded into the general case of a plane wave for any incidence angle and any polarization in Ref. [29].

In the present work we used numerical methods to solve the equation governing the evolution of the light polarization in Jones' matrix formalism at the same time with the reorientational Eq. (1). It gives us complete, spatial distribution of optical and director field in the LC cell. In Fig. 5a,b there are plots demonstrating ellipticity and azimuth changes obtained inside LC layer of $10 \mu \mathrm{m}$ when vector $\boldsymbol{E}$ makes initially an angle of 45 degrees with LC molecules. It is easily noticeable that the characteristics are strongly dependent on optical power. It seems that if light intensity is high enough to influence the medium, the changes introduced by the light in the molecular orientation have an effect on the light wave and 


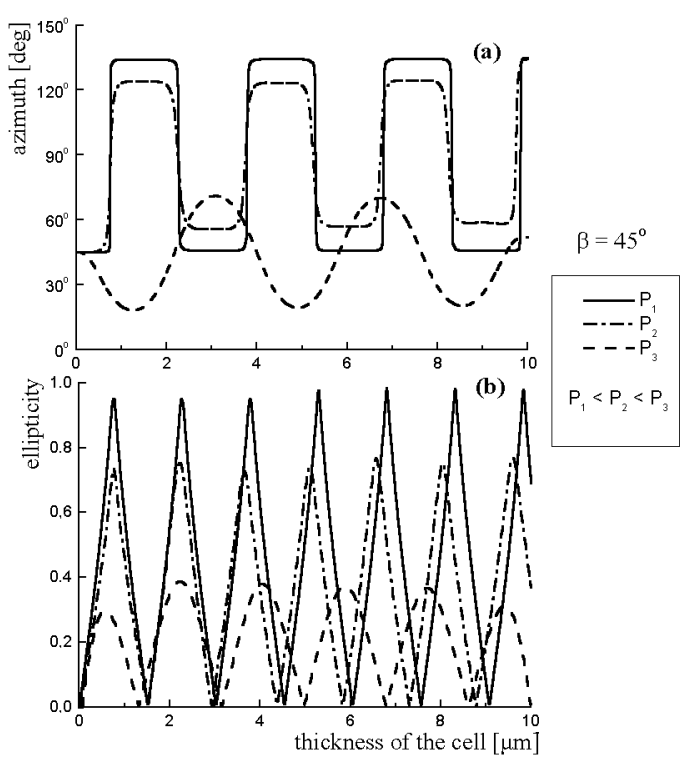

Fig. 5. Illustration of the fact how the state of polarization is changing due to nonlinear effects taking place inside LC cell. Changes of (a) azimuth and (b) ellipticity of the light propagating through the LC cell of the thickness of $10 \mu \mathrm{m}$. Initially beam is linearly polarized and vector $\boldsymbol{E}$ makes an angle of $45^{\circ}$ with director $n$.

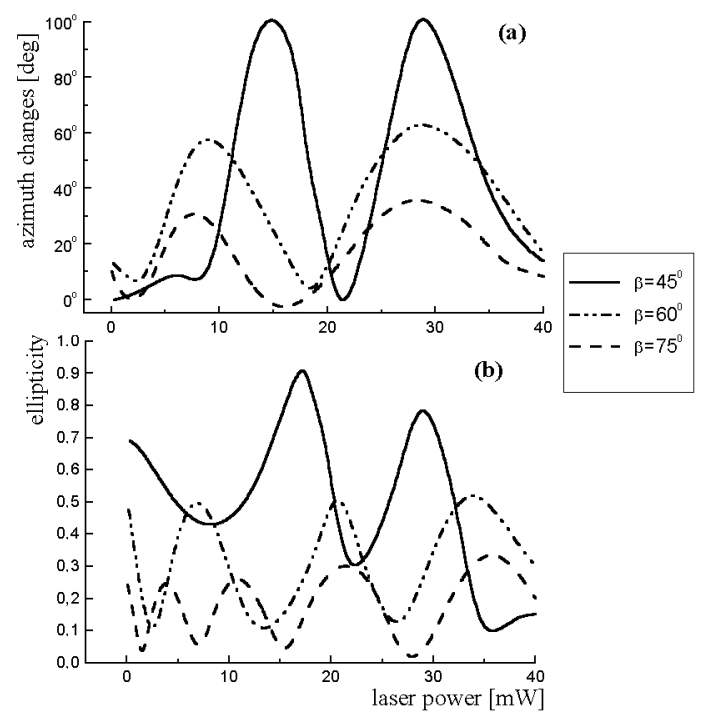

Fig. 6. Changes of azimuth (a) and ellipticity (b) of the light outcoming from the LC cell. Characteristics show dependence of nonlinear effects taking place in the sample on initial conditions. 
its polarization. As a consequence, with increasing power further outcoming from characteristics obtained for homogeneous distribution of $\theta$ (shown in Fig. 4) is observed.

In the next step, results of numerical simulations allow us to check how ellipticity and azimuth of polarization are changing after the light is passing through the LC cell. This procedure is partially determined and motivated by potential possibility of its verification and comparison with experimental results. Obtained characteristics are shown in Fig. 6a,b. They give great evidence of the fact that polarization state of the optical signal outcoming from the LC sample is strongly dependent on system geometry and light power.

\section{Experiment}

Schematic drawing of experimental setup is shown in Fig. 7. In the performed experiment 6CHBT planar nematic cell of thickness of $80 \mu \mathrm{m}$ was used. As a source of light an argon laser with wavelength of $514 \mathrm{~nm}$ and the spot size of $2 \mathrm{~mm}$ diameter was chosen. The laser beam was passing through polarizer giving TM-like polarization and then it was focused on LC cell by the lens $f=180 \mathrm{~mm}$ (according to theoretical estimation, it gives the spot size of about $25 \mu \mathrm{m}$ diameter at the sample position). By rotating the sample, the changes of the angle between input direction of electric field vector $\boldsymbol{E}$ and director $\boldsymbol{n}$ were realized. The optical power meter was used to measure the power of the output signal, i.e. the power of the light beam that came through analyzer located after cell. For fixed position of the sample the analyzer was rotated and therefore it was possible to find out how the state of polarization was changing due to nonlinear effects taking place inside examined sample.

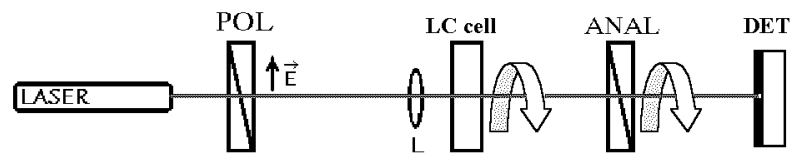

Fig. 7. Experimental setup: POL - polarizer, L - lens, ANAL — analyzer, DET detector.

As is well known, the reorientation gives rise to formation of typical self-phase-modulation rings pattern in the far field with their number depending on reorientation angle $[1-9,14]$. However, a more precise method of registration of reorientation bases on monitoring of the on-axis intensity. In our experiment, the central part of the beam (thus, inner part of diffraction pattern) was isolated due to the aperture placed in front of detector. All measurements were made in the stable stationary state and for fixed parameters no oscillations in the number of the visible rings were noticed. 


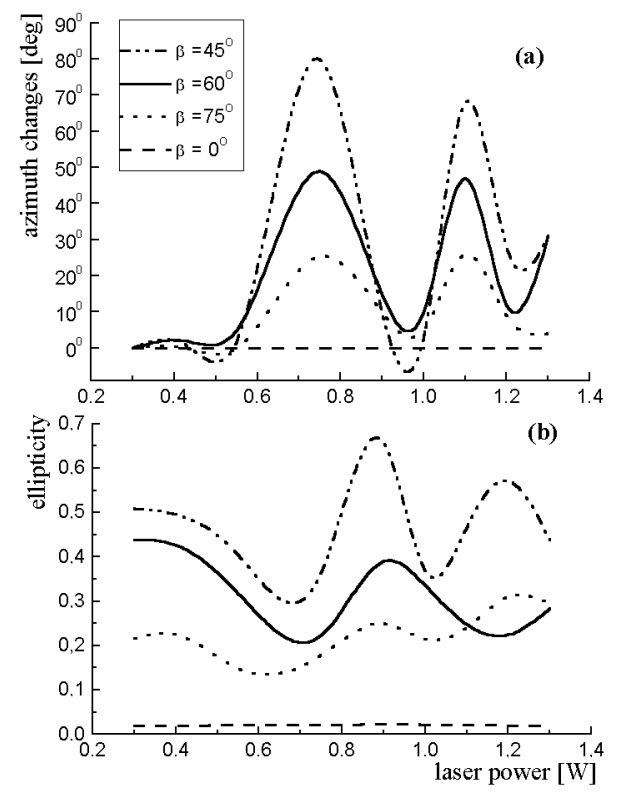

Fig. 8. Output characteristics obtained experimentally for different input polarizations (different values of angle $\beta$ ) as a function of power of light wave illuminating planar nematic LC cell: (a) azimuth changes, (b) ellipticity of the output beam.

Changes of transmitted intensity detected in the center of the outcoming beam gave us an evidence of polarization state dependence. Since input linear polarization became elliptical in the LC cell, we measured the ellipticity and azimuth changes in the center of outcoming beam with respect to the incoming power and direction of the input linear polarization. This method is rather simple from an experimental point of view but the introduced technique allowed us to get enough information on the variation in the polarization state of the laser beam and it could be considered as a low cost alternative to the one used in Ref. [17]. Plots shown in Fig. 8a and b present experimental results of both ellipticity and azimuth changes as a function of the light power outcoming from the laser. The obtained results are in qualitatively good agreement with our theoretical predictions (shown in Fig. 6) and a strong influence of polarization on molecular response is pointed out. In both cases characteristics show the same trend and their qualitative comparison gives satisfying results.

\section{Conclusions}

Our theoretical studies and experimental investigations, with addition to the huge number of publications [10-29], demonstrate that under physically reasonable assumptions detailed theoretical analyses have to include fact of polarization state 
changes inside LC cell. The polarization state is an important parameter in determination of nonlinear reorientation in NLC and this idea should be considered very carefully especially now, when nonlinear optical effects are subject of very intensive research activities because of their crucial role in development of semiand all-optical devices. Nonlinear phenomena caused by molecular reorientation and the fact of their dependence on light polarization seems to be very useful in potential applications for optoelectronic functional elements. The most important and motivating aspect in practical application of NLCs is their large birefringence and also fact that reorientation nonlinearity can be induced by very low optical power in a very wide spectral band (from near UV to far IR) which includes generally used telecommunication wavelength. Due to unique nonlinear properties there are numerous potential applications of NLCs in optical communication networks. Well-defined and controlled state of light polarization is very important for proper functioning of sensing devices and data processing systems, therefore in the perspective of this article, one can consider possibilities of particular utilizing of LCs elements as polarization modulators and polarization state change devices [30].

\section{References}

[1] N.V. Tabiryan, A.V. Sukhov, B.Y. Zel'dovich, Mol. Cryst. Liq. Cryst. 136, 1 (1986).

[2] I.C. Khoo, Prog. Opt. 26, 105 (1988).

[3] S. Chandrasekhar, Liquid Crystal, Cambridge University Press, Cambridge 1992.

[4] I.C. Khoo, S.T. Wu, Optics and Nonlinear Optics of Liquid Crystals, World Sci., Singapore 1993.

[5] I.C. Khoo, Liquid Crystals: Physical Properties and Nonlinear Optical Phenomena, Wiley, New York 1995.

[6] I.C. Khoo, T.H. Liu, P.Y. Yan, J. Opt. Soc. Am. B 4, 115 (1987).

[7] L.B. Au, L. Solymar, C. Dettmann, J. Schwartz, Physica A 174, 94 (1991).

[8] W.K. Bajdecki, M.A. Karpierz, Acta Phys. Pol. A 95, 793 (1999).

[9] K.A. Brzdąkiewicz, W.K. Bajdecki, A. Kozanecka, M.A. Karpierz, Acta Phys. Pol. A 98, 183 (2001).

[10] B.Y. Zel'dovich, N.V. Tabiryan, Y.S. Chilingaryan, Sov. Phys.-JETP 54, 32 (1981).

[11] B.Y. Zel'dovich, N.V. Tabiryan, Sov. Phys.-JETP 55, 656 (1982).

[12] H.L. Ong, Phys. Rev. A 28, 2393 (1983).

[13] A.S. Zolotko, V.F. Kitaeva, N.N. Sobolev, V.Y. Fedorovich, A.P. Sukhorukov, N. Kroo, L. Csillag, Mol. Cryst. Liq. Cryst. 15, 787 (1993).

[14] G. Cipparrone, V. Carbone, C. Versace, C. Umeton, R. Bartolino, F. Simoni, Phys. Rev. E 47, 3741 (1993); G. Cipparrone, D. Duca, N.V. Tabiryan, Opt. Commun. 140, 266 (1997).

[15] V. Carbone, G. Cipparrone, C. Versace, C. Umeron, R. Bartolino, Phys. Rev. E 54, 6948 (1996). 
[16] N.V. Tabiryan, A.L. Tabiryan-Murazyan, V. Carbone, G. Cipparrone, C. Umeton, C. Versace, T. Tschudi, Opt. Commun. 154, 70 (1998).

[17] G. Cipparrone, G. Russo, C. Versace, G. Strangi, V. Carbone, Opt. Commun. 173, 1 (2000).

[18] G. Russo, V. Carbone, G. Cipparrone, Phys. Rev. E 62, 5036 (2000).

[19] E. Santamato, B. Daino, M. Romangoli, M. Settembre, Y.R. Shen, Phys. Rev. Lett. 57, 2423 (1986); 61, 113 (1988).

[20] L. Marrucci, G. Abbate, S. Ferraiuolo, P. Maddalena, E. Santamato, Phys. Rev. A 47, 4859 (1992).

[21] E. Santamato, P. Maddalena, L. Marruci, B. Piccirillo, Mol. Cryst. Liq. Cryst. 25, 357 (1998); 328, 479 (1999).

[22] E. Brasselet, T.V. Galstian, Opt. Commun. 186, 291 (2000).

[23] E. Brasselet, B. Doyon, T.V. Galstian, L.J. Dubé, Phys. Lett. A 299, 212 (2002).

[24] E. Santamato, G. Abbate, P. Maddalena, Phys. Rev. A 38, 4323 (1988).

[25] E. Santamato, G. Abbate, P. Maddalena, L. Marrucci, Y.R. Shen, Phys. Rev. Lett. 64, 1377 (1990).

[26] L. Marrucci, F. Vetrano, E. Santamato, Opt. Commun. 171, 131 (1999).

[27] L. Marrucci, P. Maddalena, G. Arnone, L. Sirleto, E. Santamato, Phys. Rev. E 57, 3033 (1998).

[28] E. Santamato, G. Abbate, P. Maddalena, Phys. Rev. A 36, 2389 (1987).

[29] E. Santamato, G. Abbate, P. Maddalena, L. Marrucci, Phys. Rev. E 52, 5053 (1995).

[30] T.R. Woliński, A.W. Domański, M.A. Karpierz, M. Sierakowski, Optoelec. Rev. 9, $274(2001)$. 